\title{
Evaluating the use of immersive interactive mixed reality (IMR) technology in special needs education in Singapore
}

\author{
Shawn Zheng Kai Tan (D) $1, *,{ }^{\dagger}$, Maria Shendyapina $\mathbb{D D}^{2,{ }^{\dagger}}$ and Lilian Nguan Sim \\ Tay $^{1, *}$
}

${ }^{1}$ Digital Dream, Singapore and ${ }^{2}$ Human Communication, Development, and Information Sciences (CDIS), Faculty of Education, the University of Hong Kong, Hong Kong SAR

* For correspondence: STZK: shawntanzk@outlook.com; LNST:lilian@digitaldream.com.sg

${ }^{\dagger}$ These authors contributed equally

\begin{abstract}
The use of immersive interactive mixed reality (IMR) technologies has been of interest to educators due to the rapid advancement of technology, lowered cost, and increased engagement of the senses. This is particularly attractive in special needs education (SNE) as it can provide a safe space in which students can learn and practice skills needed in the real world. 86 students with diagnosed cognitive disabilities (aged 7-18) and 10 teachers from three schools in Singapore participated in the study. The Immersive Interactive Mixed Reality (IMR) was added to the school curriculum for 4-8 months, depending on the school. The efficacy and practicality of IMR intervention were assessed with qualitative interviews and quantitative surveys being analysed through statistical modelling. We showed improvement in students' mental wellbeing, academic and social skills as well as a positive effect on teachers' work satisfaction and sense of teacher efficacy. Based on the teachers' feedback, IMR was helpful for SNE because of its engaging environment, interactive and playful nature leading to higher involvement, facilitation of social skills, calming and relaxation effect, and "sandbox" mode for skills to be practiced in a safe space. The IMR limitations included overstimulation effects on certain students. Also, some activities can be performed more effectively using other tools. Overall, we present data that shows benefits and highlights the practicality of IMR in SNE and make the argument for their further usage and development.
\end{abstract}

Key words: Immersive Interactive Mixed Reality; Special Needs Education; Virtual Reality; Intellectual Disability

\section{Introduction}

With the increased accessibility to technology and rapid technological growth in recent years, the use of digital devices has been increasingly embraced by the education sector [1]. Among them, immersive technologies have been of particular interest due to the rapid technological development in visualization and interactions, together with lowered cost, making them an attractive learning aid [2]. Immersive technologies refer to forms of perceptual and interactive hardware technologies that blur the line between the physical world and the simulated or digital world. For an educator, this allows a combination of visually immersive spatial representations of data with our vestibular and proprioceptive senses [3], which further allows an added dimension in processing thoughts and ideas through spatial organization in the brain $[4,5]$. Indeed, immersive technologies have been shown to be significantly better than traditional teaching method in some cases $[6,7]$ and provide superior memory recall ability as compared to traditional desktop conditions [3]. This enhancement of learning has piqued the interest of researchers, organizations, and educators looking to add an extra dimension to the classroom [2]. A specific area of interest is the use of these technologies for special needs education for students with intellectual or cognitive disabilities [8]. For the purpose of this article, we will refer to SNE as special needs education with intellectual or cognitive disabilities, though it should be noted that special needs education also includes non-intellectual/cognitive disabilities (which we will not use the acronym SNE as to be able to distinguish the two).

Immersive technologies have the advantage in SNE as they can provide a wide range of educational stimuli in a safe space in which 
students can learn and practice skills and behaviours needed in the real world $[9,10]$. Transfer of this training then can be applied to the real world, which has already been shown in kitchen skills [11] and workplace tasks in sheltered factories [12]. Similarly, the use of immersive technology for SNE in children has been shown to be useful for teaching social [13] and practical cognitive skills [14,15]. The incorporation of interactive technology, which allows participation and freedom of children with special needs [16], can further enhance engagement in education, making them highly attractive as tools for SNE.

The extended reality can be presented via the Head-mounted display (HMD) or Wearable Immersive Virtual Reality (WIVR) allowing full visual immersion with stereoscopic vision within the virtual environment [17]. Although, it has been considered less suitable for the SNE due to the side-effects of physical discomfort [18]a projection-based Immersive Interactive Mixed Reality (IMR), such as Cave Automatic Virtual Environment (CAVE) [19] has been shown to be preferable for children with learning disabilities, e.g. that caused by Autism Spectrum Disorder (ASD), as it allows simultaneous interaction with other users and better integration with the physical world with an unobstructed view of one's own body [17]. The research on the specific use of CAVE in SNE for schoolchildren showed improvements in communication therapy [20], emotion expression and regulation, and everyday skills [14]. It has been also successfully used for neurotypical school education [21,22], although less is known about the CAVE effects on academic performance of children with special educational needs.

While apparent from the above-cited studies, much research has been done on this area, a major challenge has been that research has mostly been qualitative and perceived to be "messy", leading to low levels of acceptance by the mainstream scientific community [16]. Furthermore, these studies tend to originate in western countries, leaving a major question as to the efficacy beyond western cultures. Therefore in this paper, we used a combination of quantitative method in the form of surveys, analysed through statistical modelling, with a qualitative method in the form of interviews with teachers, to evaluate the efficacy and practicality in the implementation of immersive technologies in SNE. The CAVE IMR was used in multiple SNE schools in Singapore to examine its efficacy on psychological well-being, academic performance and participation in social life. We further examined the practicality of implementations through surveys and interviews of the teachers that used the IMR. Overall, we provide data that show the efficacy and practicality of implementing such technologies for SNE in Singapore.

\section{Methods}

\section{Participants and data collection}

86 students (aged 7-18) from three different schools (School 1: 31, School 2: 34, School 3: 21; names of school not listed to maintain anonymity), and 10 teachers participated in the study. While disabilities of students varied (included autistic spectrum disorder, Down syndrome, and general intellectual disability), they all had a commonality of intellectual or cognitive disabilities. To maintain full anonymity, only summary demographics were obtained and presented here. School 1 comprised of students with mild intellectual disabilities, school 2 comprised of students with moderate intellectual disabilities. School 3 was unable to provide diagnosed intellectual disabilities and only provided data based on the level of support needed which ranged from low $(n=2)$ to moderate $(n=11)$ and high $(n=8)$. Of the 10 teachers who participated in the survey and interview, 9 teachers had between 11 and 20 years of experience in teaching students with Special Needs, while 1 had less than 5 years of experience in the field. As the activities did not affect normal education practices (the use of IMR was already planned by the schools), and were not likely to adversely impact students' opportunity to learn the required educational content, or assessment of educators who provide instruction, and the investigators did not participate in the activities being observed, ethics approval by IRB was exempted. All parts of the study were conducted in English.

The survey design was done using the 'Social Outcomes Measurement Toolkit', a toolkit to measure the social impact of disability support which has been developed by the National University of Singapore Institute of System Science as part of the Tote BoardEnabling Lives Initiative (TB-ELI) [23]. Surveys were conducted in 3 stages (pre, mid, and post). The full timeline can be found in Supplementary Figure 1. All data was anonymised (except for school as individual schools were analysed separately) before analysis. Surveys on students were administered by teachers, while surveys on teachers were administered by an external third-party organization (Empact Shared Services Pte Ltd, Singapore) in order to minimize biases in reporting.

\section{Immersive Interactive Mixed Reality (IMR)}

The IMR setup was done in a $3 \mathrm{~m} \mathrm{X} 6 \mathrm{~m}$ (school 1, 2) or $3 \mathrm{~m} \mathrm{X} \mathrm{3m}$ (school 3) room. Projections were done on 3 walls and the floor using ultra short throw projectors (either Benq MW864UST or ViewSonic PS700oW) with each $3 \mathrm{~m}$ surface using 1 projector (school 1 \& 2: 5 projectors, school 3: 4 projectors). Wall sensors used a combination of IR sensors and camera through a 4th Generation Finger Touch Portable Interactive Whiteboard (Gloview FP4 Board; Shanghai EASI Computer Technology, China), while floor projections used a 3D tracking camera (Astra; Orbbec, MI, USA). Interactive technology used allowed for multiple interactions and multiple students to use the system together. Schools were provided with 20 templates (Supplementary Figure 2) and 80 default scenes from which the teachers could edit or create as many contents as they would require. Content was developed using a proprietary authoring tool which uses a drag and drop system and requires no coding knowledge (screenshot can be found in Fig 1A). Teachers were trained in three 2 hour sessions which covered use of software and content development. Subsequently, all content used in this study was developed by teachers. The templates were integrated by teachers as a part of the learning curriculum with specific lesson objectives. Examples include interactive elements of the Hungry Caterpillar story to support the storytelling for language classes (Fig 1B), a money game in which notes were flashed on screen and students had to choose the correct amount when prompted by teachers for numeracy (Fig $1 \mathrm{C}$ ), and simulated road crossing for life skills (Fig 1D). IMR was also used to stimulate a location, in which a teacher could bring students on a virtual tour. The schools also used IMR for leisure and relaxation where they played calming music and displayed nature scenes for students to focus on. Although the choice of the interactive scenes varied, all schools used the following categories of educational content: orientation in the city environment and social behaviour ("life skills"), numerical skills, and literacy/verbal skills. IMR sessions were conducted onsite, 30 mins once a week, with full class participation. To optimise the experience for students to interact with the IMR, most teachers limited the number of students using the IMR in each round to approximately 5 to 6 students. In bigger classes with more students, this meant that students had to learn to wait for their turn in order to use the IMR. The full list and description of IMR that schools used can be found in Supplementary Figure 3.

\section{Short Warwick-Edinburgh Mental Wellbeing Scale}

The data for psychological well-being were collected using the Warwick-Edinburgh Mental Wellbeing scale (WEMWBS) that was developed to assess the mental well-being of a general population [24]. In this study, the short WEMWBS (SWEMWBS) was used, 
Table 1. Modified Short Warwick-Edinburgh Mental Wellbeing scale. Left column shows the original statement in the Short Warwick-Edinburgh Mental Wellbeing scale, while right column shows the modified statement used in order to make it easier for the student's comprehension

\begin{tabular}{lr}
\hline Original Statement & New Statement \\
\hline I've been feeling optimistic about the future & I feel happy about growing up \\
I've been feeling useful & I feel I can help my friends \\
I've been feeling relaxed & I feel calm \\
I've been dealing with problems well & I can solve my own problems \\
I've been thinking clearly & I think before I do something \\
I've been feeling close to other people & I have good friends \\
I've been able to make up my mind about things & I can decide what I want to do \\
\hline
\end{tabular}

$\mathbf{A}$

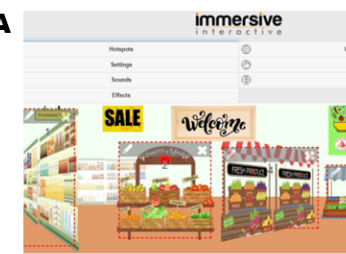

C

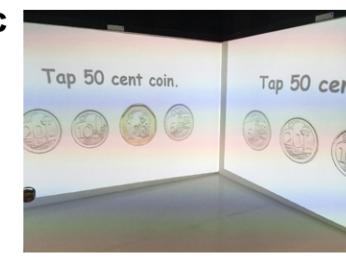

B
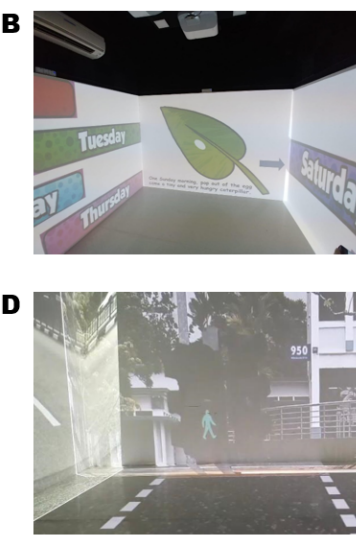

Figure 1. Examples of immersive interactive mixed reality. (A) shows an example screenshot of the drag and drop software used to develop content. (B) shows the Hungry Caterpillar story used for language classes. (C) shows a money game used to teach numeracy. (D) shows a simulated road crossing.

that has been shown to be consistent with the long-form $[25,26]$. Data was collected and reported by teachers who directly interviewed the students of their class using the given questionnaire. The statements were reworded (Table 1) to make it easier for the student's comprehension. The responses were on a 5-point scale ranging from 'none of the time' to 'all of the time' except for school 3 which opted to use a 3-point scale.

\section{Academic Performance and Social Skills}

The data on academic performance and participation in the social life of the students were collected by their teachers through observations. For both data collection, students were rated on a 5-point scale, ranging from "very poor" to "very good", except for school 3 which opted to use a 3-point scale.

Data for academic performance was based on three subjects, Literacy or Language, Numeracy, and Life Skills. The students were assessed for the change in the level of performance in these subjects using a Likert scale on the statement "Pupil acquired the intended skills [in the lesson]". Only school 1 and 2 participated in this, while no data on academic performance was collected by school 3. Data for participation in social life was observed based on three areas of communication, turn-taking and lesson engagement, using a checklist of statements. The outcome indicators for this assessment include, (i) "Pupil can communicate with others", (ii) "Pupil can wait for his/her turn", and (iii)"Pupil is engaged with the lesson". Students were assessed for the change in these three levels of participation in social life. Students in Schools 2 and 3 were assessed for all three indicators, while students from School 1 were assessed for all but lesson engagement.

Teachers' work satisfaction and sense of efficacy

The quantitative data on teachers' work satisfaction and sense of efficacy were collected using two surveys, 9-item Utrecht Work Engagement Scale (UWES) [27] and 12-item Teachers' Sense of Teacher Efficacy Scale [28] respectively. The surveys were adapted by the evaluators and administered to teachers via Google Forms. For work satisfaction and engagement, teachers were asked to indicate the change in their level of well-being and engagement after the implementation of IMR in the classroom. The questions were under categories of vigour, dedication and absorption. Responses were on a 5-point Likert scale.

For sense of efficacy, they were asked to indicate the change in their confidence after the implementation of IMR in the classroom. The questions were on the level of efficacy in student engagement, instructional strategies, and classroom management. The responses were on a 5-point scale, with 1 being "Significantly worse" and 5 being "Significantly better".

\section{Qualitative interviews with teachers}

Interviews with 10 teachers were conducted to obtain further information on their observations of students during lessons using the IMR, and to understand the teachers' experience with using the technology, as well as the benefits and challenges they faced. The interviews were conducted via online video conference calls.

\section{Statistics}

All statistical analysis was performed using R 4.0.2. Multiple time point surveys were analysed by linear mixed models (LMM) using lme4 package [29], and further analysed using lmerTest package [30], that uses Satterthwaite's method for approximating degrees of freedom for the $t$ and $F$ tests, to obtain $p$-values. Holm adjusted Tukey posthoc was used to identify individual differences in three time-point surveys. Single time point survey (UWES and sense of efficacy) was analysed using one-sample t-test with the alternative hypothesis being true mean $>3$ (which indicates a significantly positive change). Data visualizations were performed through the "ggplot2" package [31]. The level of significance for all analyses was $\mathrm{p}<0.05$. Full statistics can be found in supplementary figure 4. Effect size was not calculated as there is no existing agreed upon method to calculate standard effect size due to the way that variance is partitioned in linear mixed models [32]. The use of LMMs were however based on the vastly superior ability in controlling for Type I errors than alternative approaches which are therefore more generalizable to new observations [33]. 


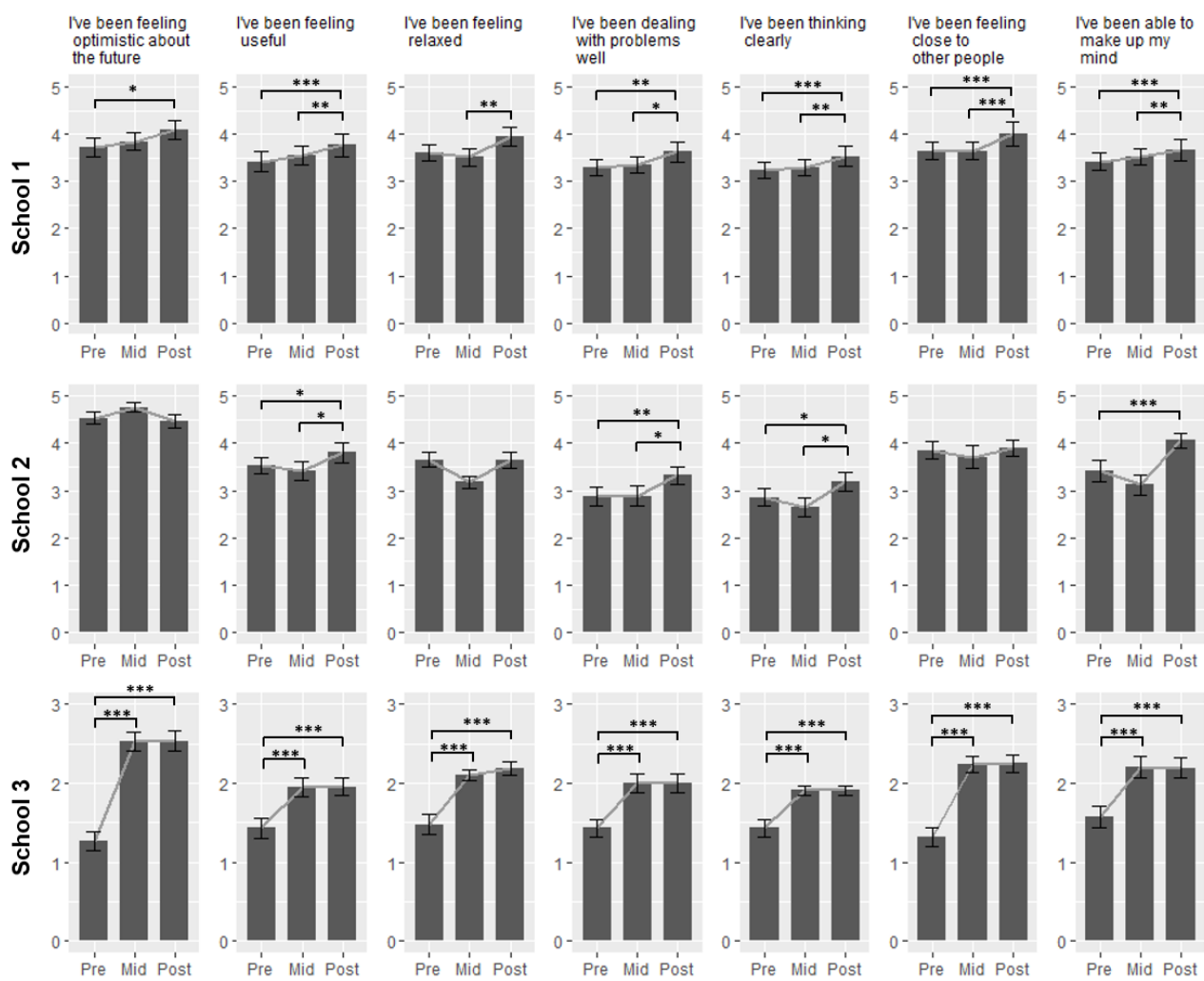

Figure 2. Immersive interactive mixed reality improves mental wellbeing in special needs education.Short Warwick-Edinburgh Mental Wellbeing scale showed improvements in all aspects in school 1 and 3, and most aspects in school 2. Data is presented as mean \pm S.E.M. of 3- or 5-point scale from 'none of the time' to 'all of the time. * refers to $\mathrm{p}<0.05, * *$ refers to $\mathrm{p}<0.01, * * *$ refers to $\mathrm{p}<0.005$.

\section{Results}

\section{IMR improves mental wellbeing in SNE}

To measure if IMR could lead to an improvement in mental wellbeing in SNE, SWEMWBS was employed pre IMR use, at a mid-point, and at the endpoint of the study (post) (Fig. 2). LMM showed significant improvements in school 1 and 3 in all aspects (lowest $\mathrm{F}$ $=4.4$, all ps $<0.05$ ). Tukey posthoc revealed that school 1 mostly showed improvements at the endpoint, while school 3 showed improvements from mid-point (though it should be noted the difference in 3-point vs 5-point scale). School 2 showed smaller effects with only 4 out of the 7 statements showing significant differences, but no significant drop regardless. Tukey posthoc similarly showed improvements at the endpoint.

\section{IMR improves academic skills in SNE}

To measure the efficacy of IMR as an educational tool for academic skills, teachers assessed students on life skills, literacy/language, and numeracy. LMM of school 1 showed significant improvement in all three areas (lowest $t=6.72$, all ps $<0.001$ ). LMM of school 2 showed significant improvement in literacy $(\mathrm{t}(31)=4.03, \mathrm{p}<$ $0.001)$, but not life skills $(\mathrm{t}(31)=1.68, \mathrm{p}=0.10)$ or numeracy $(\mathrm{t}(31)$ $=1.98, \mathrm{p}=0.06)$ (Fig. 3). Academic skills data was not available for school 3 .

\section{IMR might improve social skills in SNE}

To measure the efficacy of IMR as an educational tool for academic skills, teachers assessed students on their ability to communicate with others, wait their turn, and engage with the lesson. LMM showed significant improvements in school 1 and 3 in all aspects
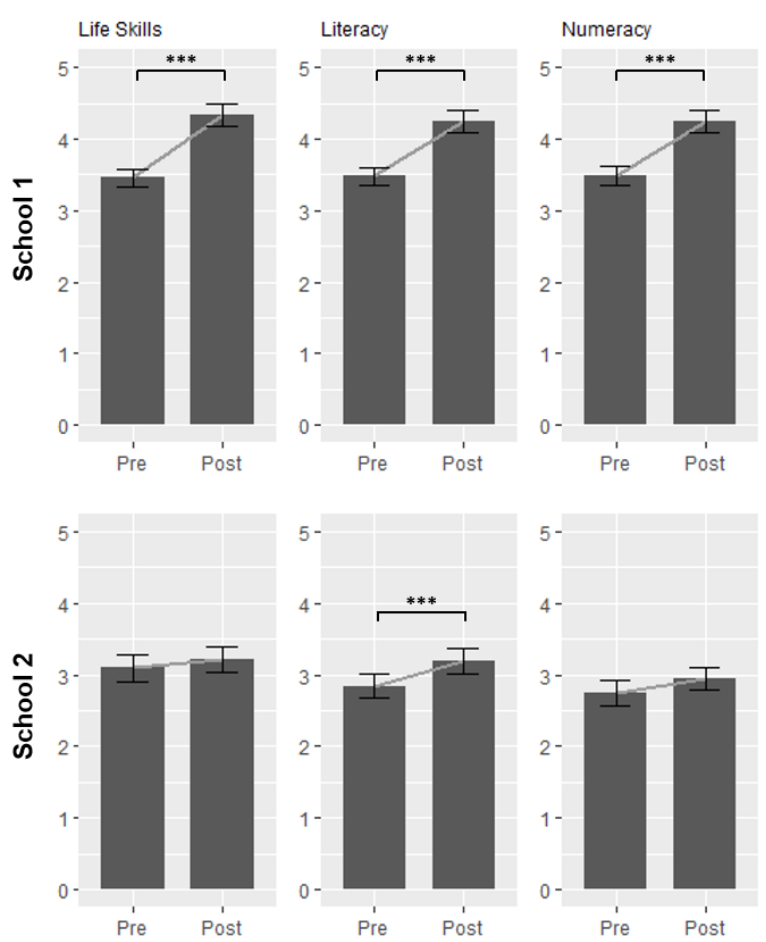

Figure 3. Immersive interactive mixed reality improves academic skills in in special needs education.Teachers assessed students on life skills, literacy/language, and numeracy pre and post immersive interactive mixed reality use. School 1 showed improvements in all aspects, while school 2 showed significant improvement only in literacy. Data is presented as mean \pm S.E.M. with 3- or 5-point scale from "very poor" to "very good". *** refers to $\mathrm{p}<0.005$. 


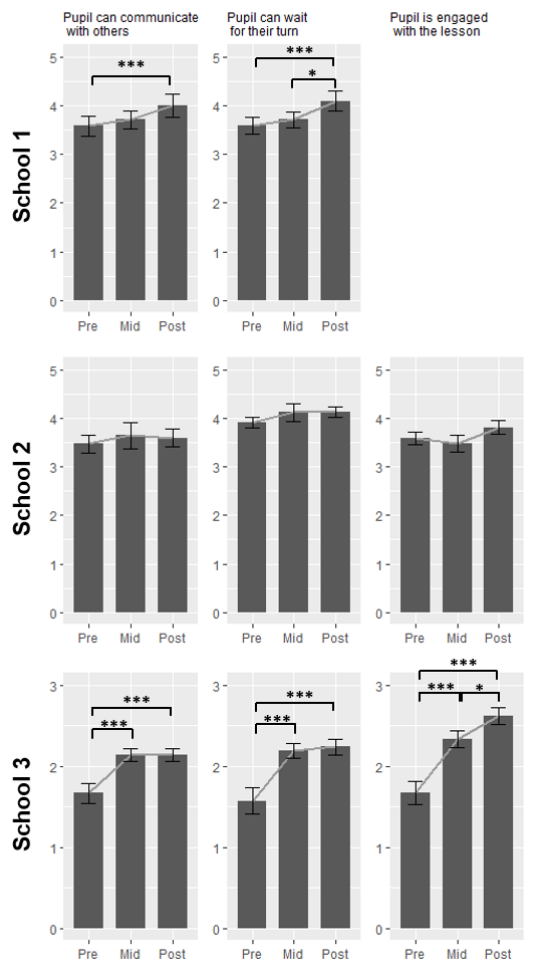

Figure 4. Immersive interactive mixed reality might improve social skills in special needs educationTeachers assessed students on social skills using a checklist of statements: (i) "Pupil can communicate with others", (ii)"Pupil can wait for his/her turn", and (iii) "Pupil is engaged with the lesson". Data is presented as mean \pm S.E.M. of 3 - or 5 -point Likert scale $*$ refers to $\mathrm{p}<0.05$, *** refers to $\mathrm{p}$ $<0.005$

(lowest $\mathrm{F}=5.2$, all ps $<0.01$ ). Similar to SWEMWBS results, Tukey posthoc revealed that school 1 mostly showed improvements at the endpoint, while school 3 showing improvements from mid-point. School 2, however, showed no significant effect on time in all three measures (highest $\mathrm{F}=2.93$, all ps $>0.01$ ) (Fig. 4).

\section{IMR has a positive effect on teachers' work satisfaction and sense of efficacy}

To understand how practical and effective IMR is in SNE, teachers responded to a 9-item UWES and 12-item Teachers' Sense of Teacher Efficacy Scale survey. One sample t-test revealed significant improvements in 4 out of 9 items in the 9-item UWES, and 10 out of 12 items in the 12-item Teachers' Sense of Teacher Efficacy Scale survey (Fig. 5)

\section{Qualitative interview with teachers}

To obtain further information on the use of IMR in SNE, interviews were conducted with teachers. The key benefits gathered from the interviews were primarily in the area of social skills. A key observation shared by teachers was that students were more engaged in lessons when using IMR, and this engagement was sustained for a longer period of time as compared to a traditional classroom setting. Some teachers shared that their students were more vocal during lessons with IMR, and some non-vocal students also made attempts to communicate:

"My students are non-verbal, and sometimes they're very tired or sleepy due to seizures or their medication. In the [IMR] room, they are looking around or they are trying to make some sounds, maybe trying to communicate. If they're not engaged they may not make any sound, or they will just look down."
One teacher explained that the immersive nature of the IMR was a possible factor to engage the students and to minimise distractions:
"One factor could be the surface area [that the IMR occupies], be- cause it's reflected on all walls and the floor as well, so [the effects] appear no matter where the students look at... In a usual classroom, students may not know where to look at or to focus on, because there are many things to look at. Other than the powerpoint, they may also look at the window..."

Several teachers also mentioned that their students were motivated and excited for the class when they had the opportunity to use the IMR. One teacher mentioned that when she asked students what they wanted to do, they would point towards the IMR room to indicate that they were hoping to have more of such sessions. Teachers also shared that student's interest and engagement levels increased with the use of IMR.

\begin{abstract}
"Some of the students who are normally very quiet or passive, with [immersive] technology, they will say that they want to play this or play that, ... [they will be] willing to take turns and demonstrate good behaviour so they get the chance to play it."
\end{abstract}

The increased classroom engagement also translated into better behaviour in class. In particular, many teachers highlighted turntaking as one such behavioural change. To optimise the experience for students to interact with the IMR, most teachers limited the number of students using the IMR in each round to approximately 5 to 6 students. In bigger classes with more students, this meant that students had to learn to wait for their turn in order to use the IMR. Teachers reported that students were generally cooperative as they were eager for their turn to use the IMR:

"I will tell them that you have to wait, so you get to choose which activity you get to do. They are able to listen to basic instructions.... because they're all looking forward to playing. By doing that, we are also teaching them to be patient, and to watch how their friends play."

The teachers highlighted the calming effect of some existing templates within the IMR system as a benefit for their students and in particular, for students with Autism Spectrum Disorder (ASD). Several teachers mentioned that their students enjoyed and responded well to the activities. The IMR classroom also provided students with an opportunity to learn in a safe and controlled environment. With the IMR, teachers mentioned that students could experience the 'real world' settings and practise new skills without being exposed to the actual environment and the risks they posed.

"IMR provides a safe environment where students could interact with their environment prior to facing the actual one. This provides a platform for the students to practise and be more ready when placed in the actual environment."

The interviewed teachers described the use of the IMR as an informal assessment method to help them determine if students have understood the instructions or concepts in lessons in a less stressful environment.
"In [SNE], many of us don't use pen and paper assessment as much as in the mainstream schools, so [the IMR] provides us a new and fun way to assess students. It might also be less stressful for students, because they don't know they are being assessed, but the teachers can tell if they are learning through the activities."

The IMR also provides a more controlled environment that allows teachers to assess students' understanding of specific concepts of skills and allows teachers to understand the students' needs.

"We did not have any platform to expose our students to certain scenarios... Some of the scenarios in the IMR provided additional data 
to understand our students' needs and anxieties better e.g. one was afraid to step on a virtual water scene."

As described above, teachers had used the IMR in different ways and for a variety of different classes and purposes. However, during the interviews, several teachers shared that they found the existing templates available on the IMR platform to be limited in range, and largely suited for leisure or relaxation activities. These teachers expressed that they hoped to see more IMR templates that were directly relevant to the syllabus or topics they were teaching in class, in order to help them achieve the relevant academic outcomes for students. Similar to results on social skills (Fig. 4), teachers, especially those whose classes were higher-functioning, reported that they did not experience much difference as they had not experienced many challenges in relation to student behaviour before the use of the IMR. In some instances, students could also become overly excited or stimulated with the IMR and become harder to manage. However, this was rare and only occurred in a few specific instances. Similarly, several teachers also reported no change in teachers' well-being with the use of the IMR. These teachers shared that they had already been actively using different tools (including digital tools) to teach their classes and to engage students. Thus, the use of IMR alone did not result in a significant change in well-being. Teachers also pointed out that their students tend to have difficulty generalising learning from IMR's artificial environment to the actual 'real-world' environment. Thus, the IMR was helpful to complement but cannot replace the actual outdoor interaction.

\section{Discussion}

In this study, we took a practical approach in order to determine both the efficacy and practicality of using IMR in SNE in Singapore. Using surveys and statistical modelling, we showed that IMR is useful as an educational tool for SNE in mental wellbeing and academic skills and might also be useful for social skills. We further showed that IMR had a positive effect on both teachers' work satisfaction and teachers' sense of efficacy, which demonstrates the practicality of the use of IMR in SNE. Lastly, interviews with teachers generally agreed with survey results, and further highlighted some challenges with the use of IMR.

The choice of allowing teachers to create content for IMR that they use was based on the concept of participatory design of which
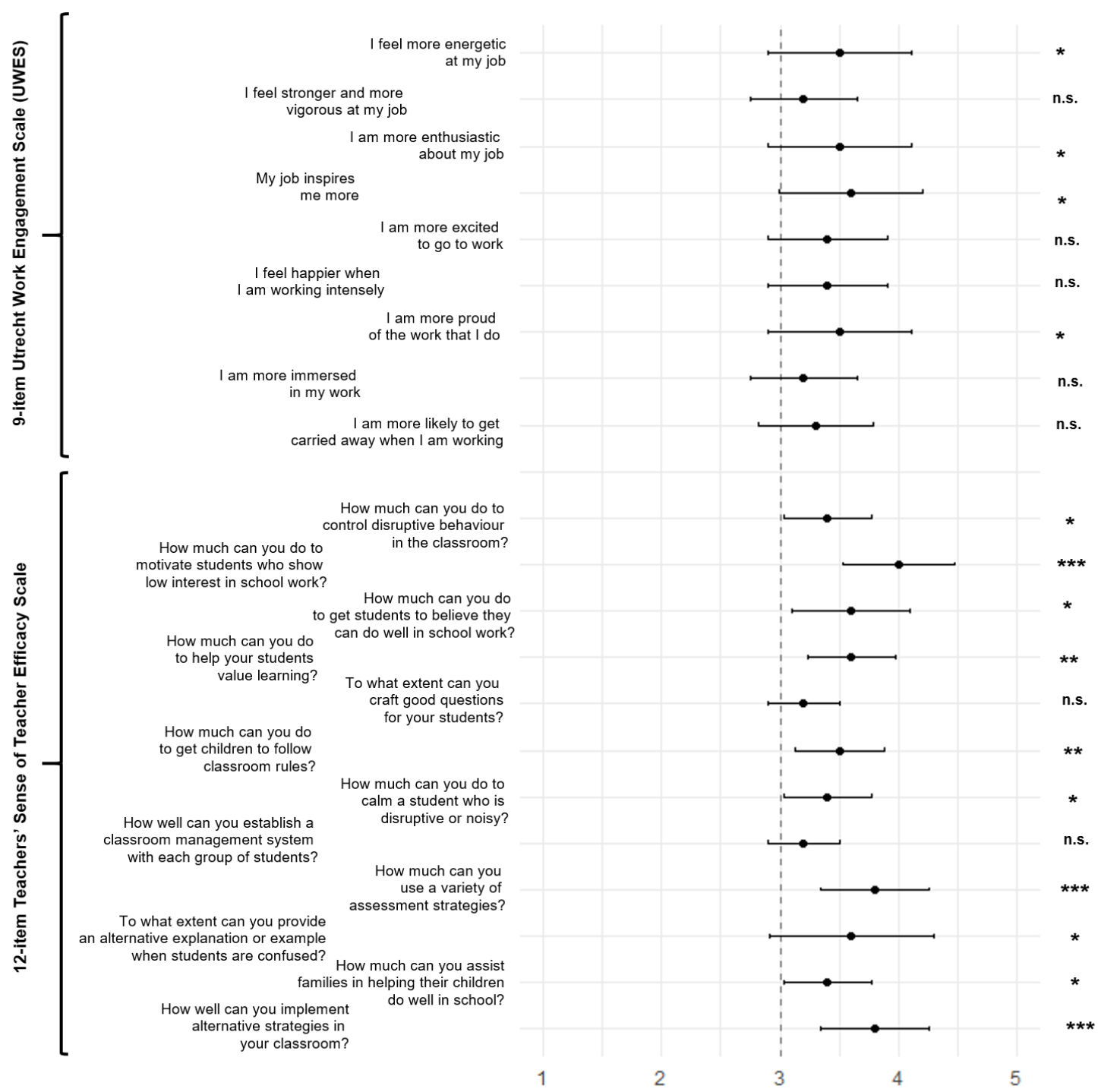

Figure 5. Immersive interactive mixed reality has a positive effect in teachers' work satisfaction and sense of efficacy.Teachers responded to a 9-item Utrecht Work Engagement Scale and 12-item Teachers' Sense of Teacher Efficacy Scale survey. Data is presented as mean $\pm 95 \%$ CI on a 5-point scale with 1 being "Significantly worse" and 5 being "Significantly better". * refers to $\mathrm{p}<0.05, * * *$ refers to $\mathrm{p}<0.005$, n.s. refers to not significantly different, $\mathrm{p}>0.05$. 
benefits have been discussed and summarized by Alper et al. [16]. This, however, comes with the challenge of training teachers to use such technologies, something we did indeed probe by surveying teachers which showed positive results (discussed later). However, we do note that in interviews, teachers highlighted the limited amount templates (20) which suggests the difficulties of designing content on the teachers' part - something that has to be taken into consideration when using participatory design in IMR. While our approach allows participatory design from teachers, more work is needed in order to allow participatory design by students too, challenges of which have been similarly discussed by Alper et al. [16]. We suggest allocation of additional training time for teachers in IMR classes design is required for more effective use of the technology. Alternatively, a series of structured educational IMR sets for the typical SNE setting with a wide range of difficulty levels could be used.

The use of a modified SWEMWBS was chosen over the full WEMWBS was done in consultation with teachers. The short version has been shown to be consistent with the long version [26] but with reduced participant burden. Given that the population of interest in this paper was students in SNE, statements of the SWEMWBS had to be edited as words such as "optimistic" and concepts such as "feeling useful" were difficult to convey to students with cognitive disabilities. The results showed improvements in students' mental wellbeing in all schools in most of the questions asked. Interestingly, however, we do find differences in patterns of increase with school 1 and 2 showing changes only in post-IMR but not mid-IMR, but school 3 showing changes by mid-point. This could be due to the difference in the scale used, the difference in activities and uses in different schools, or most likely a combination of both. Regardless, this suggests that perhaps relatively longer periods/sessions ( 9 months) are required for effects and has implications on how SNE schools should use IMR if they were to adopt it (e.g. one-off sessions might not be useful).

Students with learning disabilities (like those in SNE) often manifest social skill deficits [34] while developing social skills is crucial for academic achievement as well as work-related skills [35]. Given that immersive technology has been shown to be useful for teaching both social and cognitive skills [13,14], we decided to probe the efficacy of IMR in both by the means of teacher's evaluation. Our results show improvement in all areas in school 1, but only literacy in school 2. Similarly, for social skills, school 1 showed improvements but not school 2. Overall academic and social skills seem to show the same trend in improvement. This trend is similar to that of mental wellbeing scores with school 2 showing less pronounced effects. It is unfortunate that we were unable to obtain full sets of data, however, our results show that IMR is indeed useful in certain scenarios, although their effects vary widely from centre to centre. In interviews, teachers mentioned that students had difficulties generalising learning from IMR's artificial environment to the actual 'real-world' environment which could account for some of the limited effects seen. Overall while IMR seems to have success as a teaching tool in certain academic and social skills, the effects varied widely, and more work is needed to understand what are the best approaches to use IMR for social and academic skills. The most stable effect of IMR was found for the mental wellbeing of children which goes in line with a existing evidence on soothing and relaxing IMR effects, e.g., improving mental wellbeing of patients in pediatric oncology [36] or effective VR stress management on a workplace [37], albeit the effect could be partially caused by the music therapy which is shown to be efficient for people with ASD [38]. A relatively good IMR effect on social skills could be explained by combination of the CAVE stimuli and maximized social interaction with peers and teachers during the sessions which may lead to better social skills generalization [39]. A fewer number of academic improvements could be explained by the missing templates in the IMR system, as admitted in the teachers interview. In addition, the interac- tive framework of the CAVE seems more suitable for supplementary training of academic skills like conceptual learning [40] or illustrating learned concepts [41] rather than substituting the formal classroom. A review by [42] also suggests that VR technologies could be beneficial in middle school to complement studying complex 3D objects, so a better effect can be expected from the older SNE sample. Another study from [43] demonstrated the successful use of IMR in learning foreign language and culture where CAVE was perceived by students as a more exciting and immersive medium compared to PC, which gives a hint that IMR may indirectly improve academic performance through raising learning motivation. This factor can be recommended for verification in further research.

Given that one of the major stakeholders in the use of IMR in SNE are the teachers, we wanted to understand how IMR affects their job and their sense of it - for IMR to be practically implemented, it is crucial that teachers using it both sense its efficacy and are not burdened by it. Indeed, teachers with a stronger sense of efficacy tend to show better planning and organization [44], and are more open to new ideas and methods (such as IMR) to better meet the needs of their students $[45,46]$. Our results showed that using IMR increased engagement in teachers ( 4 out of 9 items in the 9 item UWES), and increased their sense of teacher efficacy (10 out of 12 items), overall showing that teachers do indeed see the benefits of IMR in SNE. This is echoed in the interview with teachers sharing anecdotal stories on how IMR has helped their students. To sum up, IMR was helpful for SNE because of its (1) engaging environment preventing the students from distraction; (2) interactive and playful nature of classes leading to higher involvement and motivation of the students; (3) facilitation of social skills via the need to take turns in the games; (4) calming and relaxation effect; (5) "sandbox" mode for different skills which could be informally assessed and practiced in a safe space. At the same time, the IMR had certain limitations, such as (1) underutilization of templates due to the limited time frame of training; (2) overstimulation effect on certain students; (3) some activities can be performed more effectively using other tools.

Interestingly, our study is not the first in Singapore to probe the efficacy of using an interactive immersive virtual environment for SNE. Lu et al. [47] similarly showed beneficial effects of IMR with children with autistic spectrum disorder. However, their study was limited by small sample size $(n=12)$ and limited scope. Our work therefore both validates and extends their findings.

\section{Limitations}

While our aim in this paper was to provide robust data on the efficacy and practicality of the use of IMR in SNE in Singapore, we acknowledge a few limitations that should be noted when making conclusions from this paper. Similar to Lu et al. [47], our work is limited by missing samples and a still relatively small sample size. Furthermore, given the broad scope of our work, we were unable to fully delve into the nuances of each of the benefits seen. A major limitation to our experimental design is the lack of a control group. However, due to the opportunity cost of a control group (half the SNE students not being able to use IMR), comparing preIMR to post-IMR seemed a more feasible way, even compared to a counter balanced cross-design (which would require more manpower and time). Regardless, future work that looks a more well controlled experimental design could be useful in determining the actual efficacy of IMR in comparison with more traditional methods of SNE. Lastly, due to the sensitive nature of SNE settings and a preliminary nature of this specific intervention, a lot of potentially informative data was not available. Experiments and correlative analysis of the impact of participants demographics (age, gender, socioeconomic status, level of disability, and diagnosis), teachers experience and abilities (level of the IMR design proficiency, atti- 
tude towards technology) could provide further insight into what the optimal strategy for the IMR implementation in SNE would be. Furthermore studies looking at standardized educational content would greatly aid in variance in data, and overall provide better insight into the technology.

\section{Conclusions}

In this study, we demonstrated with both quantitative and qualitative methods that IMR in SNE is beneficial to students' mental wellbeing, acquisition of social and academic skills, and teachers' work engagement and sense of efficacy. Overall, we present a strong argument on the further development of IMR for SNE. More work is however needed to fully understand the nuances of our results if we are to more effectively utilize IMR in SNE. We are however optimistic about the potential of this technology to improve and aid current SNE.

\section{Acknowledgements}

This work was funded by grants from SG Enable's Tote Board Enabling Lives Initiative ("TBELI") Grant awarded to Digital Dream. We would like to thank Empact for their valuable input.

\section{Declaration of Conflicting Interests}

LNST and SZKT are affiliated with Digital Dream, a company that provides IMR solutions and related services. Empact, which did some data collection and interviews, were hired by Digital Dream to conduct said surveys and interviews.

\section{References}

1 Zawacki-Richter O, Latchem C. Exploring four decades of research in Computers \& Education. Comput Educ. 2018;122: 136152.

2 Radianti J, Majchrzak TA, Fromm J, Wohlgenannt I. A systematic review of immersive virtual reality applications for higher education: Design elements, lessons learned, and research agenda. Comput Educ. 2020;147: 103778.

3 Krokos E, Plaisant C, Varshney A. Virtual memory palaces: immersion aids recall. Virtual Real. 2019;23: 1-15.

4 Knauff $M$. Space to reason: A spatial theory of human thought. Mit Press; 2013

5 Roediger HL III. Implicit and explicit memory models. Bull Psychon Soc. 1979;13: 339-342.

6 Dede C, Salzman MC, Bowen Loftin R. ScienceSpace: virtual realities for learning complex and abstract scientific concepts. Proceedings of the IEEE 1996 Virtual Reality Annual International Symposium. IEEE; 1996. doi:10.1109/vrais.1996.490534

7 Salzman MC, Dede C, Loftin RB, Chen J. A model for understanding how virtual reality aids complex conceptual learning. Presence . 1999;8: 293-316.

8 Cobb SVG. Virtual environments supporting learning and communication in special needs education. Top Lang Disord. 2007;27: 211-225.

9 Standen PJ, Brown DJ. Virtual reality in the rehabilitation of people with intellectual disabilities: review. Cyberpsychol Behav. 2005;8: 272-82; discussion 283-8.

10 Wallace S, Parsons S, Westbury A, White K, White K, Bailey A. Sense of presence and atypical social judgments in immersive virtual environments. Responses of adolescents with Autism Spectrum Disorders. Autism. 2010;14: 199-213.

11 Rose F, Brooks BM, Attree E. Virtual reality in vocational training of people with learning disabilities. 2000
12 Mendozzi L, Pugnetti L, Barbieri E, Attree E, Rose F, Moro W, et al. VIRT - factory trainer project. A generic productive process to train persons with disabilities. 2000 [cited 2 Oct 2020].

13 Matsentidou S, Poullis C. Immersive visualizations in a VR Cave environment for the training and enhancement of social skills for children with autism. 2014 International Conference on Computer Vision Theory and Applications (VISAPP). 2014. pp. 230-236. 14 Tzanavari A, Charalambous-Darden N, Herakleous K, Poullis C. Effectiveness of an immersive virtual environment (CAVE) for teaching pedestrian crossing to children with PDD-NOS. 2015 IEEE 15th International Conference on Advanced Learning Technologies. IEEE; 2015.

15 Cromby JJ, Standen PJ, Newman J. Successful transfer to the real world of skills practised in a virtual environment by students with severe learning difficulties. for Disability, Virtual .... 1996.

16 Alper M, Hourcade JP, Gilutz S. Interactive technologies for children with special needs. Proceedings of the 11th International Conference on Interaction Design and Children - IDC '12. New York, New York, USA: ACM Press; 2012.

17 Ip HHS, Wong SWL, Chan DFY, Byrne J, Li C, Yuan VSN, et al. Enhance emotional and social adaptation skills for children with autism spectrum disorder: A virtual reality enabled approach. Computers and Education. 2018;117: 1-15.

18 Garzotto F, Gelsomini M, Occhiuto D, Matarazzo V, Messina N. Wearable Immersive Virtual Reality for Children with Disability: a Case Study. Proceedings of the 2017 Conference on Interaction Design and Children. New York, NY, USA: Association for Computing Machinery; 2017. pp. 478-483.

19 Cruz-Neira C, Sandin DJ, DeFanti TA. Surround-screen projection-based virtual reality: the design and implementation of the CAVE. Proceedings of the 2oth annual conference on Computer graphics and interactive techniques. New York, NY, USA: Association for Computing Machinery; 1993. pp. 135-142.

20 Bryant L, Brunner M, Hemsley B. A review of virtual reality technologies in the field of communication disability: implications for practice and research. Disabil Rehabil Assist Technol. 2020;15: $365-372$.

21 Roussou M. Immersive interactive virtual reality and informal education. Proceedings of User Interfaces for All: Interactive Learning Environments for Children. 2000. pp. 1-9.

22 Roussou $M$, Oliver $M$, Slater $M$. The virtual playground: an educational virtual reality environment for evaluating interactivity and conceptual learning. Virtual Real. 2006;10: 227-240.

23 Greulich-Smith T. Measuring the Social Impact of Disability Support. 22 Mar 2017 [cited 9 Oct 2020]. Available: https://www.iss.nus.edu.sg/community/newsroom/newsdetail/2017/03/22/measuring-the-social-impact-of-disabilitysupport

24 Tennant R, Hiller L, Fishwick R, Platt S, Joseph S, Weich S, et al. The Warwick-Edinburgh Mental Well-being Scale (WEMWBS): development and UK validation. Health Qual Life Outcomes. 2007;5: 63.

25 Stewart-Brown S, Tennant A, Tennant R, Platt S, Parkinson J, Weich S. Internal construct validity of the Warwick-Edinburgh Mental Well-being Scale (WEMWBS): a Rasch analysis using data from the Scottish Health Education Population Survey. Health Qual Life Outcomes. 2009;7: 15.

26 Ng Fat L, Scholes S, Boniface S, Mindell J, Stewart-Brown $S$. Evaluating and establishing national norms for mental wellbeing using the short Warwick-Edinburgh Mental Well-being Scale (SWEMWBS): findings from the Health Survey for England. Qual Life Res. 2017;26: 1129-1144.

27 Schaufeli WB, Bakker AB, Salanova M. The measurement of work engagement with a short questionnaire. Educ Psychol Meas. 2006;66: 701-716.

28 Tschannen-Moran M, Hoy AW. Teacher efficacy: capturing an elusive construct. Teach Teach Educ. 2001;17: 783-805.

29 Bates D, Mächler M, Bolker B, Walker S. Fitting lin- 
ear mixed-effects models Usinglme4. J Stat Softw. 2015;67. doi:10.18637/jss.v067.io1

30 Kuznetsova A, Brockhoff PB, Christensen RHB. LmerTest package: Tests in linear mixed effects models. J Stat Softw. 2017;82. doi:10.18637/jss.v082.113

31 Wickham H. ggplot2: Elegant Graphics for Data Analysis. Springer-Verlag New York; 2016.

32 Rights JD, Sterba SK. Quantifying explained variance in multilevel models: An integrative framework for defining R-squared measures. Psychol Methods. 2019;24: 309-338.

33 Barr DJ, Levy R, Scheepers C, Tily HJ. Random effects structure for confirmatory hypothesis testing: Keep it maximal. J Mem Lang. 2013;68.

34 Kavale KA, Forness SR. Social skill deficits and learning disabilities: a meta-analysis. J Learn Disabil. 1996;29: 226-237.

35 Lynch SA, Simpson CG. Social Skills: Laying the Foundation for Success. Dimensions of Early Childhood. 2010;38: 3-12.

36 Tennant M, Youssef GJ, McGillivray J, Clark T-J, McMillan L, McCarthy MC. Exploring the use of Immersive Virtual Reality to enhance Psychological Well-Being in Pediatric Oncology: A pilot randomized controlled trial. European Journal of Oncology Nursing. 2020. p. 101804.

37 Naylor M, Ridout B, Campbell A. A Scoping Review Identifying the Need for Quality Research on the Use of Virtual Reality in Workplace Settings for Stress Management. Cyberpsychol Behav Soc Netw. 2020;23: 506-518.

38 Geretsegger M, Elefant C, Mössler KA, Gold C. Music therapy for people with autism spectrum disorder. Cochrane Database of Systematic Reviews. 2014. doi:10.1002/14651858.cdoo4381.pub3

39 Mesa-Gresa P, Gil-Gómez H, Lozano-Quilis J-A, Gil-Gómez JA. Effectiveness of Virtual Reality for Children and Adolescents with Autism Spectrum Disorder: An Evidence-Based Systematic Review. Sensors . 2018;18. doi:10.3390/s18082486

40 Johnson A. VR as Instructional Technology-The CAVE as Classroom. Configuring history: Teaching the Harlem renaissance through virtual reality cityscapes Retrieved November. 2006;12: 2008.

41 Sun J, Li H, Liu Z, Cai S, Li X. An empirical case on integration of immersive virtual environment into primary school science class. Proceedings of 25th international conference on computers in education (ICCE). 2017. pp. 566-575.

42 Freina L, Ott M. A Literature Review on Immersive Virtual Reality in Education: State Of The Art and Perspectives. 2015.

43 O'Brien MG, Levy R, Orich A. Virtual immersion: The role of CAVE and PC technology. Calico Journal. 2009.

44 Allinder RM. The Relationship Between Efficacy and the Instructional Practices of Special Education Teachers and Consultants. Teacher Education and Special Education. 1994;17: 86-95.

45 Guskey TR. Teacher efficacy, self-concept, and attitudes toward the implementation of instructional innovation. Teach Teach Educ. 1988;4: 63-69.

46 Stein MK, Wang MC. Teacher development and school improvement: The process of teacher change. Teach Teach Educ. 1988;4: 171-187.

$47 \mathrm{Lu} \mathrm{A}$, Chan S, Cai Y, Huang L, Nay ZT, Goei SL. Learning through VR gaming with virtual pink dolphins for children with ASD. Interact Learn Environ. 2018;26: 718-729. 